و إمكانية الاستفادة منها في
دور الضرائب البيئية في الحد من التلوث ـ

كلية الادارة والاقتصاد

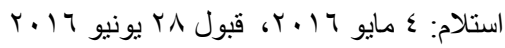

ان مبدأ الضر ائب البيئية يعتمد على ان الذي يحدث ررا بيئيا هو من يدفع ضر ائب اكثر ، وذلك كعقوبة على تدمير البيئة

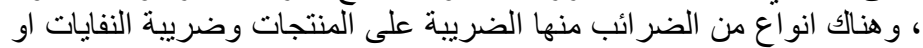

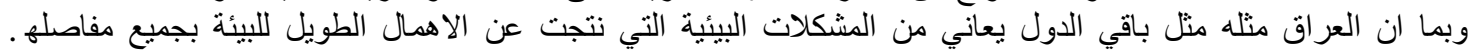

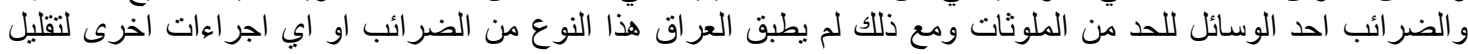

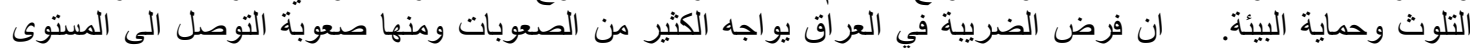
ثالي للضر ائب الحكومية لحماية البيئة و وغيرها.

الكلمات المفتاحية: الضر ائب البيئية، نلوث البيئة، النفايات، حماية البيئة، الموارد الطبيعية، التغير المناخي.

$$
\begin{aligned}
& \text { ضية الدراسة: } \\
& \text { نطلق الدر اسة من الفرضية التالية التالية: }
\end{aligned}
$$

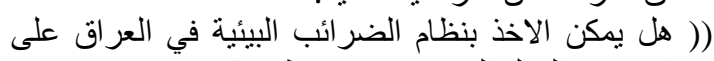

$$
\begin{aligned}
& \text { غرار بعض الدول التي نجحت في تطبيقها )) } \\
& \text { هدف الاراسة: } \\
& \text { - توضيح ماهية الضر ائب البيئية وكيفية تحديدها. } \\
& \text { - ما هي الدول التي طرقت باب الضرائب البيئية ونجحت } \\
& \text { في تطبيقها. } \\
& \text { : } \\
& \text { السياسات هي الضر ائب البيئية. } \\
& \text { : مفهوم ونشأة الضر ائب البيئية: } \\
& \text { النظام الضريبي البيئي: }
\end{aligned}
$$

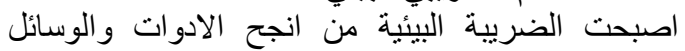

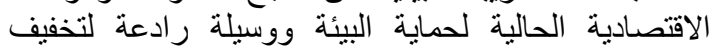

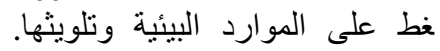

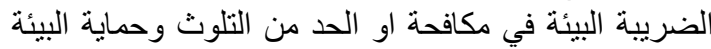

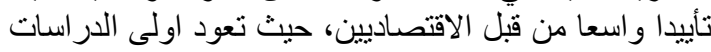

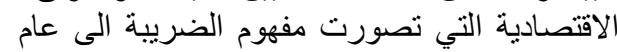
وذلك عندما نشر الاقتصادي البريطاني سييل بيغون في في كتابه

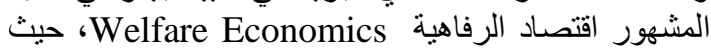
التأثنير

$$
\text { الانتاج او الاستهلاك. }
$$

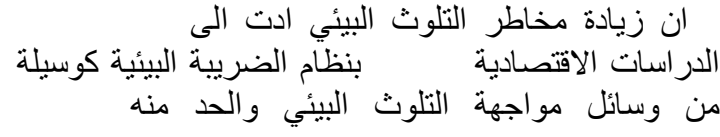

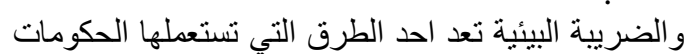

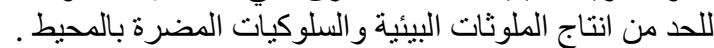

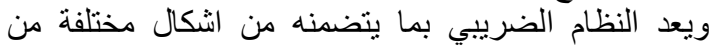

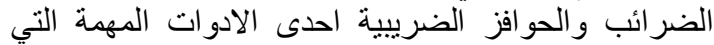

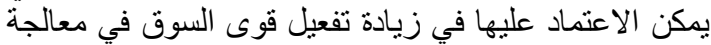

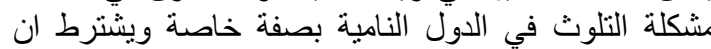
تكون الحصيلة الضريبية الضون

حيث كانت اهم المبررات هي حماية البيئة من التلوث اد على التقليل من التلوث البيئي

ان الضريبة البيئي

من الملوثين في مقابل منحهم حق استعمال وتلويث الفيثة البيئة.

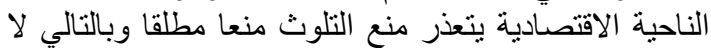

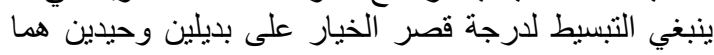

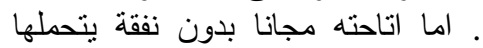
لتلوث فكل من البديلين غير و اقعي.

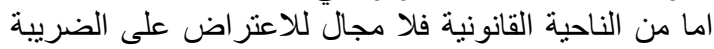

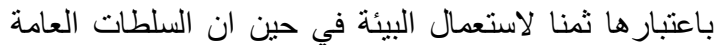

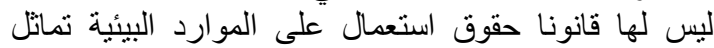
ها لا يتعدى مركز المسؤل.

* Corresponding author:

Dr. Muntder Fadil Saad

$凶$ muntadarfadhel@yahoo.com 


\section{الطريقة هذا هو الحديات المتساوية.}

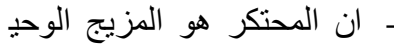

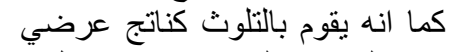

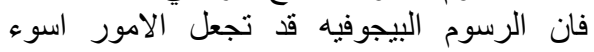

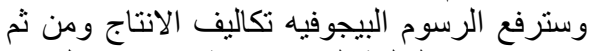
ينخفض ناتج السلعة المحتكرة اكثر من ذبي قبل ممال

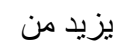

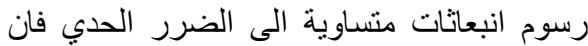

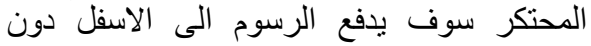

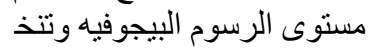

و عليه يمكن تعريف النظام الضريبي بصفة عامة على انه مجموعة القو اعد القانونية والفنبة التي تمكن فئن

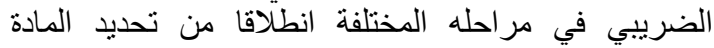

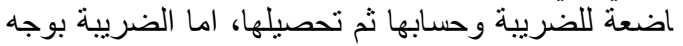
عام فهي عبارة عن اقتطاع نقدي اجباري من الافر اد مساهمة

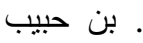

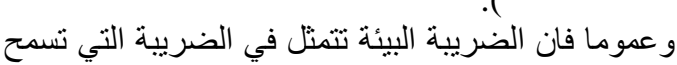
قيمة نقدية لاستغلال موارد البيئة التي غالباً ما يتم استغلالها بالمجان، بحيث لجيث نجد ان المنتجين يلقون بنفاياتهم

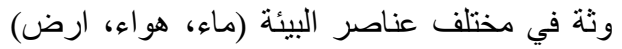
مقابل ومجال الضر ائب البيئية يرتكز اساسه على الاقتطاعات

اما بالنسبة لمنظمة التعاون والتتمية الاقتصادية فقد عرفتها بانها جملة الاجر اءات الجبائية التي يتسم و عائها بكونه ذات التهات تاثير سلبي على البيئة.

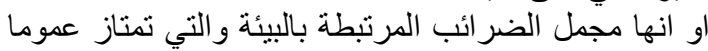

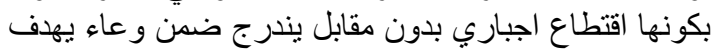
من خلاله حماية البيئة (حونيه بن طييه ، ).

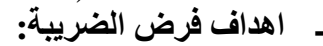

هنالك مجموعة من الآهداف التي يسعى المشروع البئئي

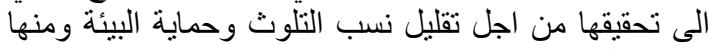

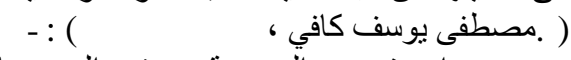
ـ - لئن فرض الضريبة يهذف الى حماية الانسان بتوفير الظروف البيئية المناسبة الخالية من كل النل مظاهر التلوث، بل ان هذا الهئية الندف كان وراء

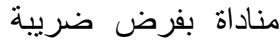
تطبيقا للقاعدة التي تقوم عليها السياسة الضريبية

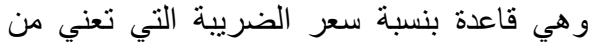

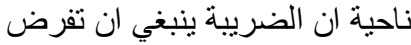
سبة الى السلع ذات الاستعمال الثنائع و هي تلألك

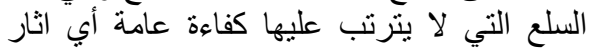

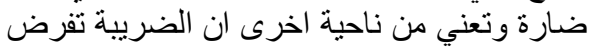

ـ ـ المساهمة في ازالة التلوث من خلال الاجراءات

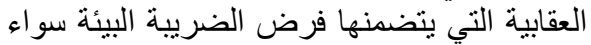
كانت غرامات مالية او عقوبات جنائية يتعرض البئية لها لها

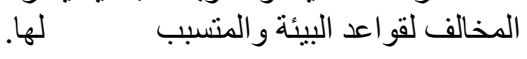

وكان الاساس العلمي في فرض الضريبة البيئية هو المبدا

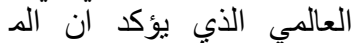

الضريبة، وقد تطرقت الهم الدراسات الاقتصادية و الاجتماعية

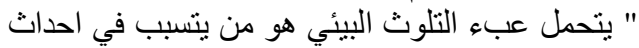

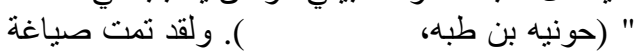

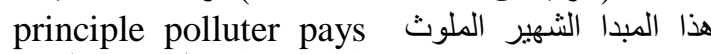
من طرف منظمة التعاون والتنمية

الاقتصادية ويعني هذا المبدا ان الملوث يجب انب ان يتحمل

$$
\text { تكاليف ومحاربتها هم الملوثون. }
$$

وعموما فان الضريبة البيئة تتمثل في الضريبة التئي تسمح

قيمة نقدية لاستغلال موارد البيئة التي غالباً ما يتم

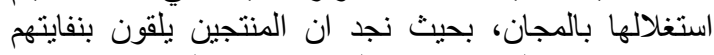

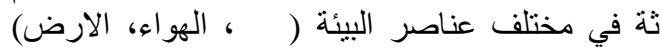

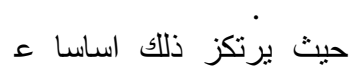

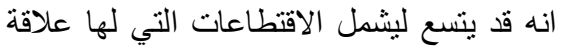

ببعض طرق تيسير الموارد الطبيعية كما ان اوعية الاقتطا

في ضوء ذلإك نم اللجوء اللى وسائل التحفيز الضريب

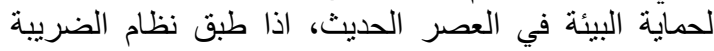

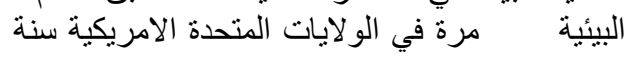

tax expenditure

جزئي او كلي من دفع الرسوم الايكولوجية اذذا امتتلت التئل

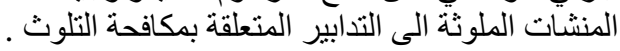

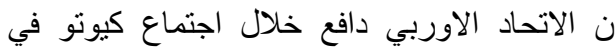

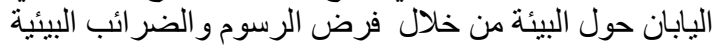

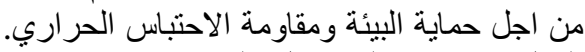

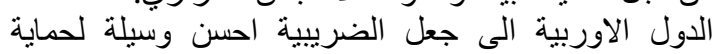
البيئة، مما ادى الىى ان تصبح هذه الطريقة افضل وسيلة الثية

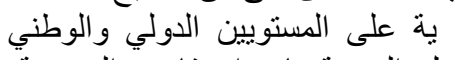

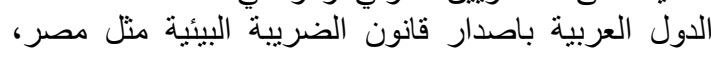

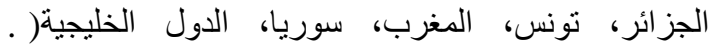
مصطفى يوسف كافي، تونئ - مفهوم الضر ائب البيئية: القد عرفت الضر الثب البيئية

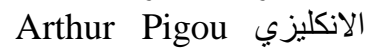
الاجتماعية للتاني تكلفة الملوثين سالبة لأنهم يوفرون نقودا عن طريق التلوث

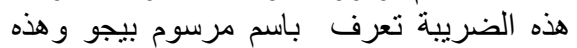
الضريبة البيئة هي ( . عبدالامير عبدف عبدالحسين شباع وقاسم

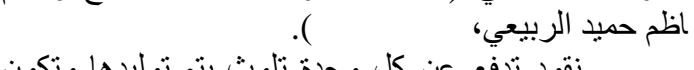
نقود تدفع عن كل وحدة تلوث يتم توليدها وتكون

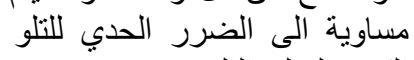
الكف ألتوليد التلوث.

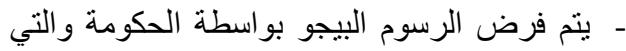
تتولى جميع ايرادات الرسوم تعمل عاد الرئل توثيق تئن ـ في حالة العديد من الملوثين تتطلب الكفاة في التحكم في التلوث وان تكون التكلفة الحدية متساوية فئية لجميع الّلوثين ولكن بشرط ان يكون اسهام 


$$
\begin{aligned}
& \text { يض التلوث عند المستوى الامثل له } \\
& \text { وكما في الثكلين التاليين: }
\end{aligned}
$$

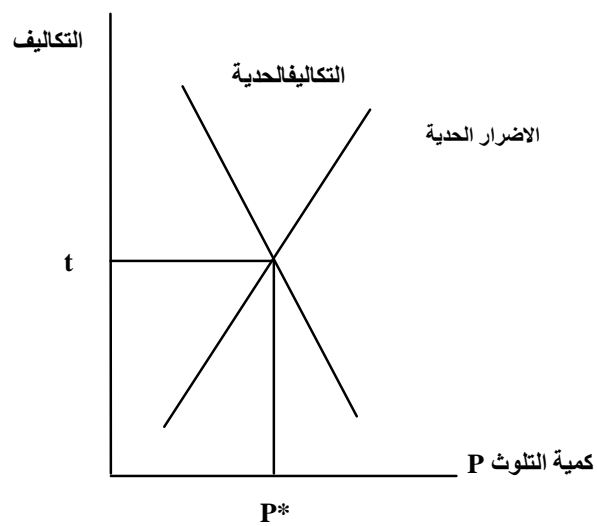

( )

Source: Kerr,S,2001,Ecological tax Reform, Report prepared for the Ministry of Environment, motu, Economic and public policy Research, Newzealand, 23 January.P57.

وفي الحقيقة ان ضريبة بيغوفيان المثالية من الناحية

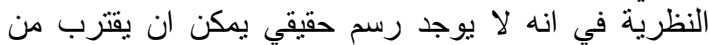
ضريية بيغوفيان الصحيحة من الناحية النظرية. الحل المثالي للتلوث و الضرائب المثالية فاننا نسعى للتحدث

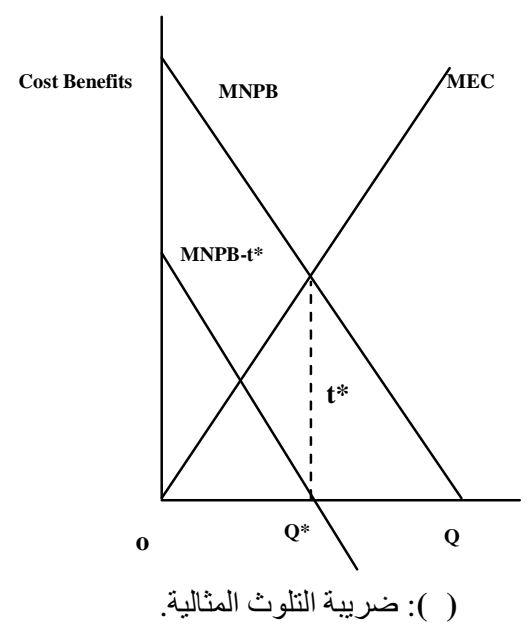

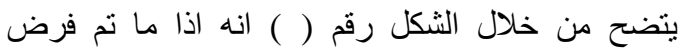

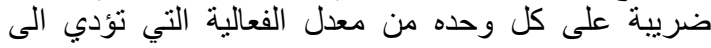

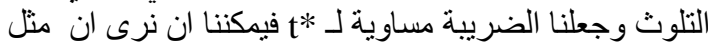
تللك الضريبة سوف يكون لها اثر تحويل MNPB

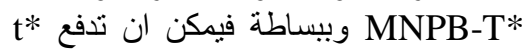

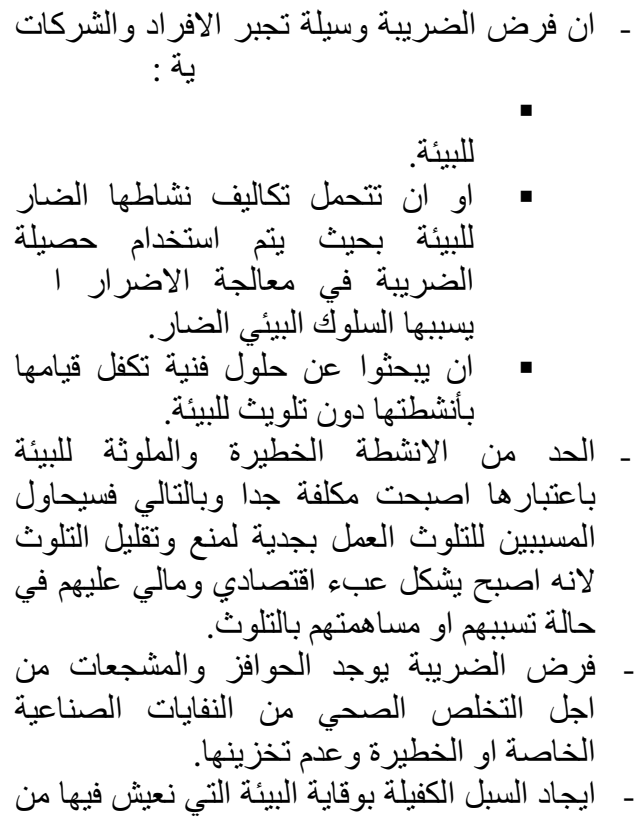

- ايجاد ونوفير المصادر المالية الجديدة لحماية

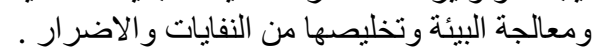

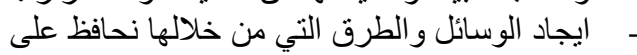

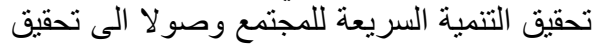
التنمية المستدامة الكفيلة بادامة المواردة للعة لاديال

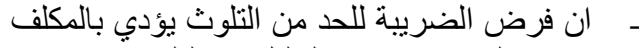

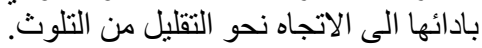

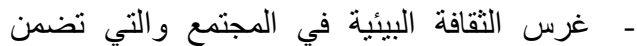

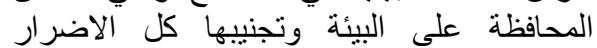

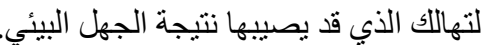

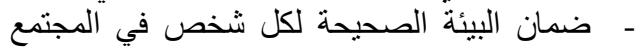

وحسب ما طرحه الاقتصادي Pigou اذن يعتبر التلوث

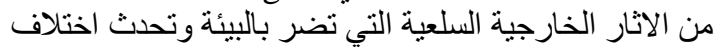

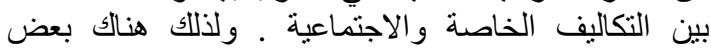

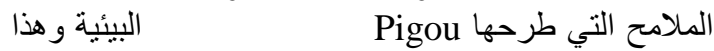

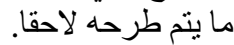

Pigou ثانياً: قياس الضر ائب البيئية: ي فرض ضريبة على الانشطة الملوثة الخارجية للتلوث، او بعبارة اخرى تلكفل تضمين التهين التكاليف الخارجية للتلوث ضمن التكاليف الخاصة للمنتجين المتسببين

وهذا الامر يؤدي الى تصحيح فثل السوق والتخصيص التص ولئور للك الحد من التلوث البيئي، ويتمحور

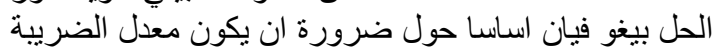

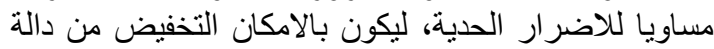

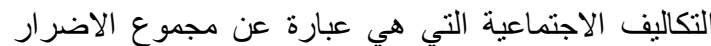

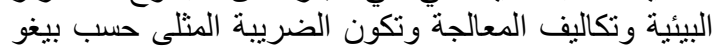




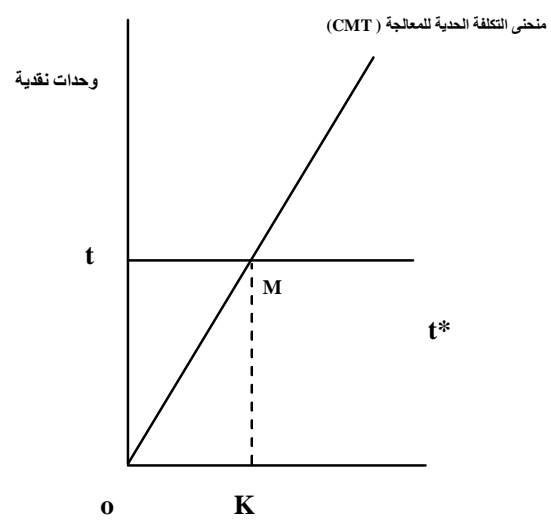

( ): السعر الامثل لضريبة التلوث بطريقة الضريبة

يتبين من الثكل اعلاه ان الضريبة ثابتة يمثلها الخط

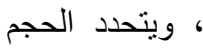

التي يتعادل عندها كل (M)

(tt*) المستقيم (t)

(k)

منحنى التكلفة الحدية لمعالجة التلوث (CMT)

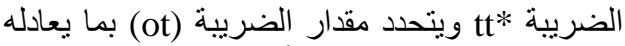

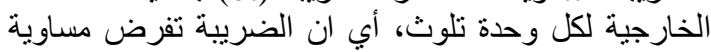
لفة الخارجية لكل وحدة تلوثة لكون.

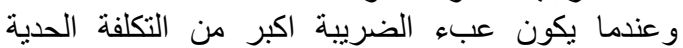

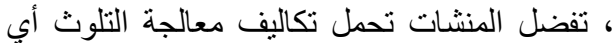

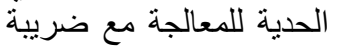

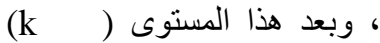

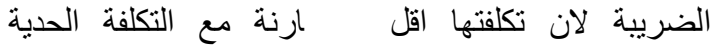
جه هذه الطريقة جملة من الصنوبات:

$$
\text { (فيزيائي، كيميائي }
$$

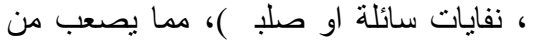

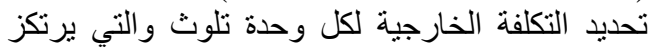
عليها في تحديد مقدار الضريبة الثانية، كما ان تحديد لكل وحدة تلوث يتوجب توفر نظام معلومات متطو وخبرة علمية و عملية قلما لوحن

اختلاف طبيعة المنشات الملوثة وكذلك تشكيلة

اختلاف الطرق والاساليب الفنية و التكنولوجية

اختلاف المواصفات الفنية لكل عنصر من من عناصر التلوث من ناحية

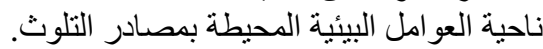

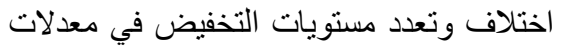
التلوث المطلوب تحقيقها خلال الفتر ات فئن الزمنية t* فعالية بحيث ينخفض صافي المنفعة الحدي بقيمة المنافي

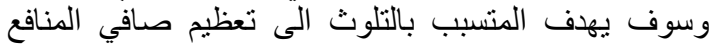

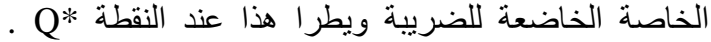

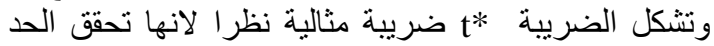
$\mathrm{Q}^{*}$

و السؤال الذي يطرح نفسه كيف يتم تحديد t* ؟ انها تساوي

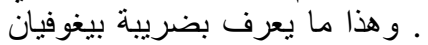

MEC

المثالية. التي تساوي التكلفة الحدية الخارجية بعرفية

وتشير دالة الضرر الى كيفية تباين التلوث مع مستوى التلوث

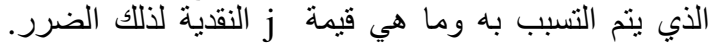

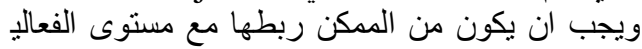
يقوم المتسبب بالتلوث بكن من العان

MEC و هذا يعني اننا بحاجة لإيجاد بل بلاد

\section{()}

: تحديد سعر ضريبة التلوث.

من المعلوم ان الاسعار او المعدلات الضربـ الضريبية، اما ان تكون

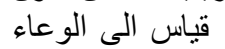

ضل استخدام وتطبيق اسلوب

الذي تستند عليه.

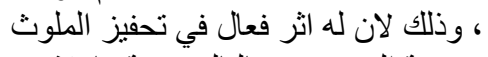

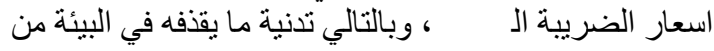

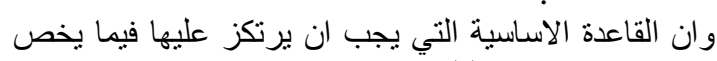

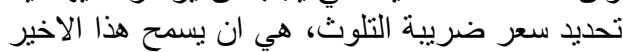
التكاليف الخارجية للتلوث.

ويمكن استخدام سعر ضريبة التلوث كاداة تمييزية بحيث

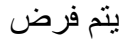

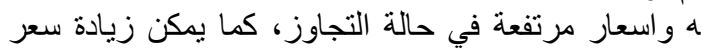

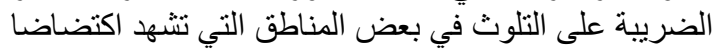

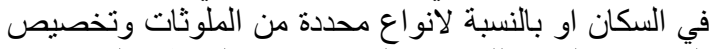

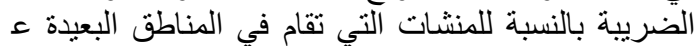

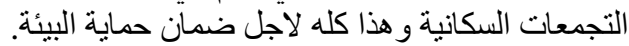

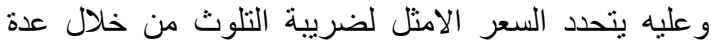

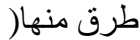

لحالة المبسطة لفرض ضريبة

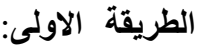

، بحيث تكون هذه الاخيرة ثابتة وتفرض على لفى كل

النظر عن اجمالي كمية التلوث، ويبين ونين التالي كيفية تطبيق هذه الضريبة. 


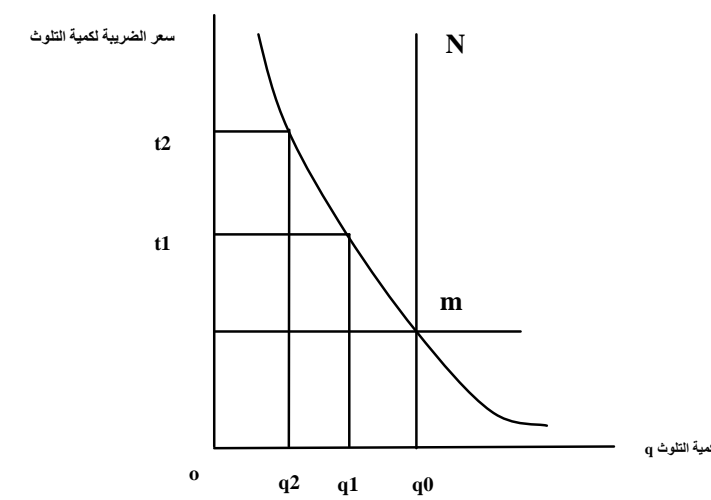

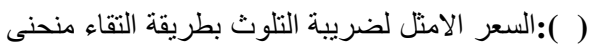

تكلفة المعالجة مع المعيار المحدد للتنلوث المسموح بـه.

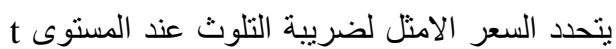

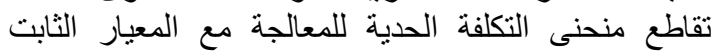
المحدد من قبل الجهات البيئية

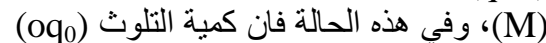

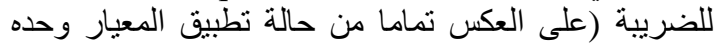

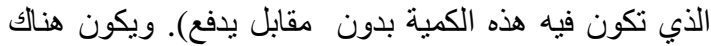

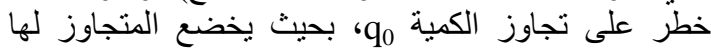

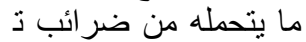

فيمتها تكاليف معالجة التلوث. وتمكن هذه الطريقة الجهات المتخصصة من نحريك سعر الضريبة فيكانية التي قد تثنه كثافة سكانية عالية

الملوثات شديدة الخطورة بهدف تدنية كمية التلوث وتحقيق اية تعديلات العلت

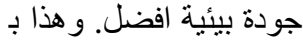

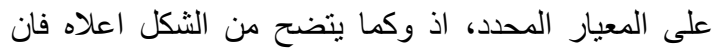

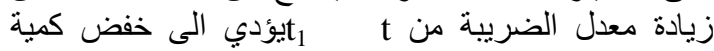
$t_{2} t_{2} q_{2} \quad q_{1}$

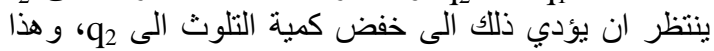
بالنظر الى تكلفة الضريية في هذه الاحو ال التي لئي ، مما يجبر الملوث على تلنية حجم الاحية

$$
\text { التلوث الناشئ عن نشاطه الانتاجي. }
$$

وتعد هذه الطريقة انسب الطرق لتقدير سعر الضريبة و هذا

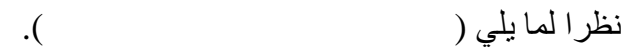

- عدم الاحتياج لكم هائل من المعلومات والمعطيات و الإمكانيات لتقدير اضرار التلوث كما هو الحال في

ـ ـ المزاوجة بين طريقة التنخل المباثرة والمعيار المحدد

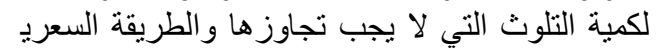
المعتمدة على فرض ضرية لاتية التلوث، مما يؤدي الى الى نجاعه بيئة افضل.

) و التي لا يشكل وجود الضريبة سواء

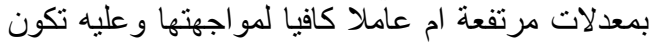

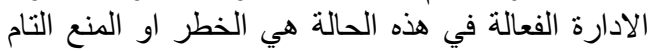

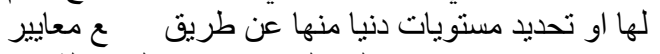

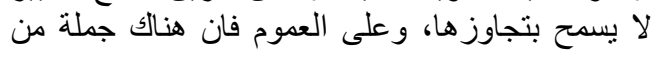

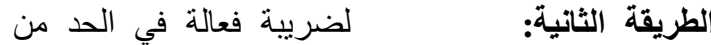

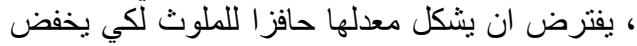

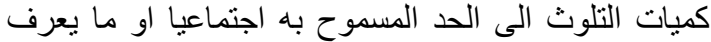
لتلوث وكما مبين في الثكل النالي:

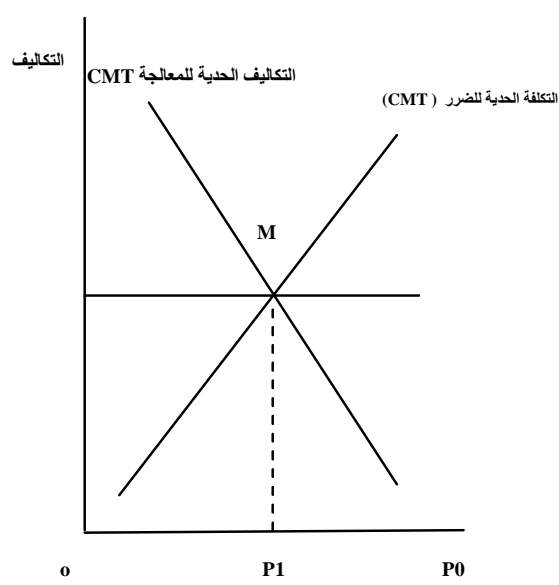

:( )

يتحدد معدل الضريبة على التلوث عند تقاطع منحنيات كل

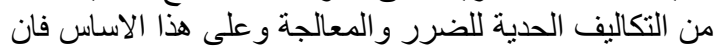

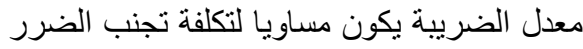

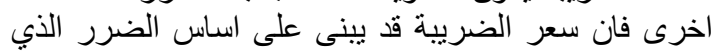

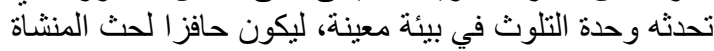

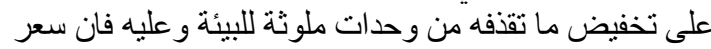

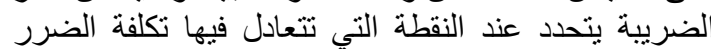

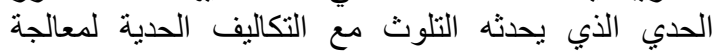

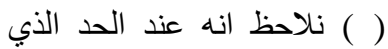

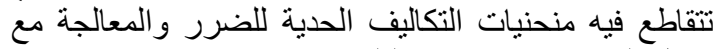
Pعد الضريبة تكون قيمة التلوث قد خفضت من

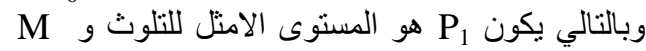
الضريبة الامتل وعند هذا تكون الضريبة قد حققت هدف لت الت النسبب يتحمل مسؤولية

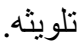

و هذه الطريقة ايضا تنطوي على صعوبات ترتبط اساسا

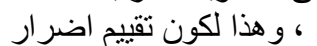
كل التقييم النقدي التطي

التلوث بتطلب معلُومات ومعطيات وكذللك امكانيات غالبا ما لكا تكون غير متو افرة.

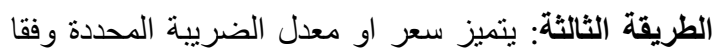

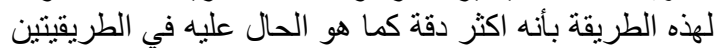
السابقتين، هذا وحسب هذه الطريقة يتم تحديد السعر الامثل للضريبة للفية عند نقطة التقاء منحنى التكلفة الحدية للمعالجة (CMT)

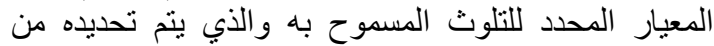
طرف الجهات المختصة بحماية البيئة و الرسم البياني التنالي يوضح ذلك. 


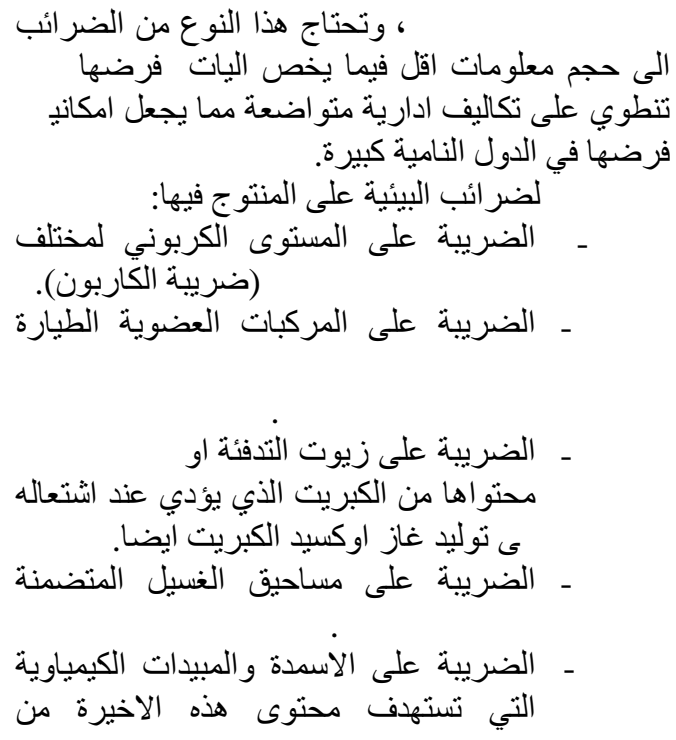

للاستفادة من خدمات بيئية معينة مثل التوصيل بشبكة

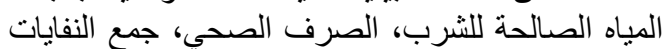

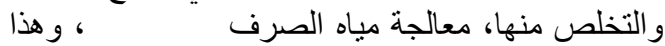

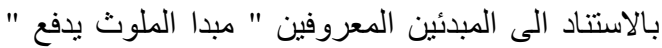

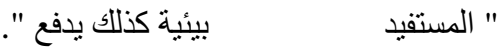

و عليه فانه يتوجب على مستخدمي البيئة المساهمة في لئي دفع وتغطية تكاليف معالجة التلّي ثلث وحماية البيئة

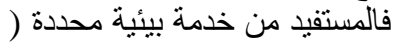

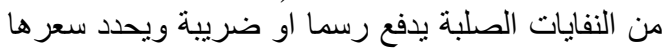

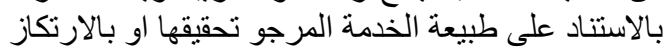
على الاهداف التي تخصص لها لخعة اير ادات هذه الضر ائب

ويعتمد هذا النوع من الضرائب على جمع ومعالجة الفضلات الاكثر تطبيقا في العديد من الدول وهي تنطلب

$$
\text { للنفايات وكذللك بعض التو النقامل النوعية كتركيز }
$$
الب على استغلال الموارد الطبيعية:

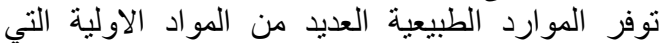
تستعمل في مختلف الانشطة وهي غالبا ما تكون مملوكة الطي

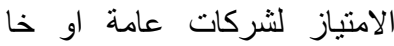

$$
\begin{aligned}
& \text { التجاري لهذه الموارد }
\end{aligned}
$$

كالغابات والثروة الموارد السمكية او الموارد غير المتجددة

لهذا يمكن تكييف الضرائب على الاستغلال التجاري للموارد الطبيعية لتحقيق اهداف بيئية الى جانب الإني الاهداف

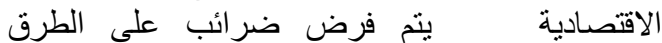
الاستغلالية للموارد الطبيعية الاكثر تلويثا وهذا بغية

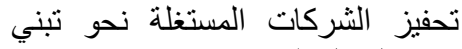
استغلال اقل تلويث.
الاعتبار ات التي يجب اخذها بالحسبان عند وضع سعر الضريبة على التلوث و المتمثلة فيما يلي: بلئ باني ضرورة ان يتسم سعر الضريبة بالمرونة الكافية " ان بر اعي عند وضع الاسعار الضريبية التمييز بين مختلف الحالات والظروف التي قد نزيد من

و الظروف التي تخفف من جسامة هذه الاضرار،

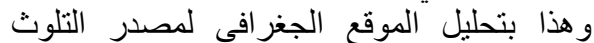

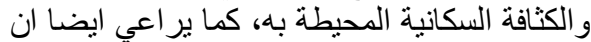
يكون سعر الضريبة تمييزيا بين

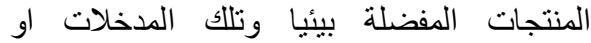
المنتجات الاخرى البديلة لها ذات الضرد البئي البئي الكبير.

: الضكال الضرائب البيئية:

تنطوي الضرائب البيئية على الثكال عديدة تهدف في البئي

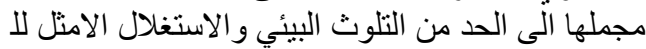

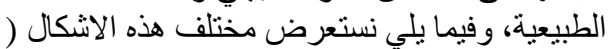

\section{:}

هذا النوع من الضرائب هو عبارة عن اقتطاع نقدي

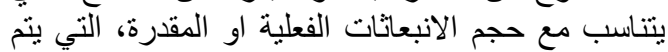

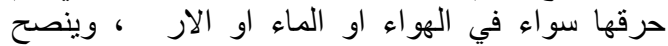
باعتماد هذا النوع من الضرائب في حالة الة الأ

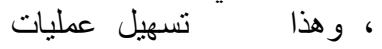

$$
\text { قبة و التسيير على الصعيد الاداري. }
$$

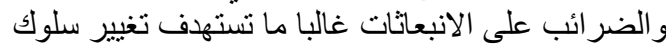

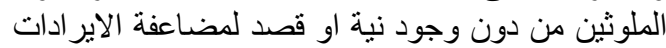

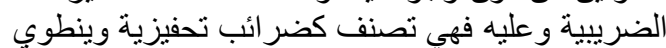

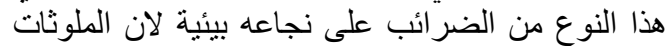

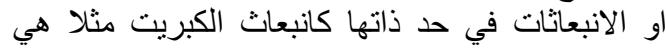
هذا النوع من الضرائب

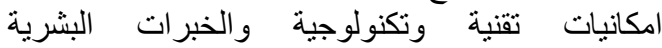

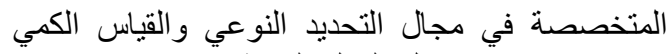

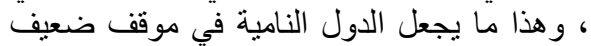

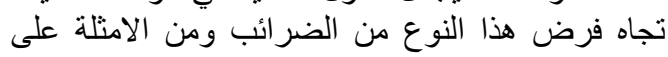
$\left(\mathrm{So}_{2}, \mathrm{No}_{\mathrm{x}}, \mathrm{co}, \mathrm{cfc}\right)$ حجم انبعاث هذه الملوث الناثشئة عنها.

ات او التخلص منها، يفرز فذإن لغة على الصحة او نفايات وملوثات، فان هذه التهان

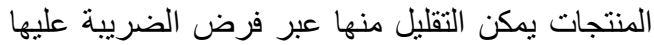

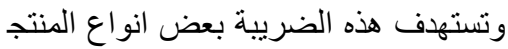




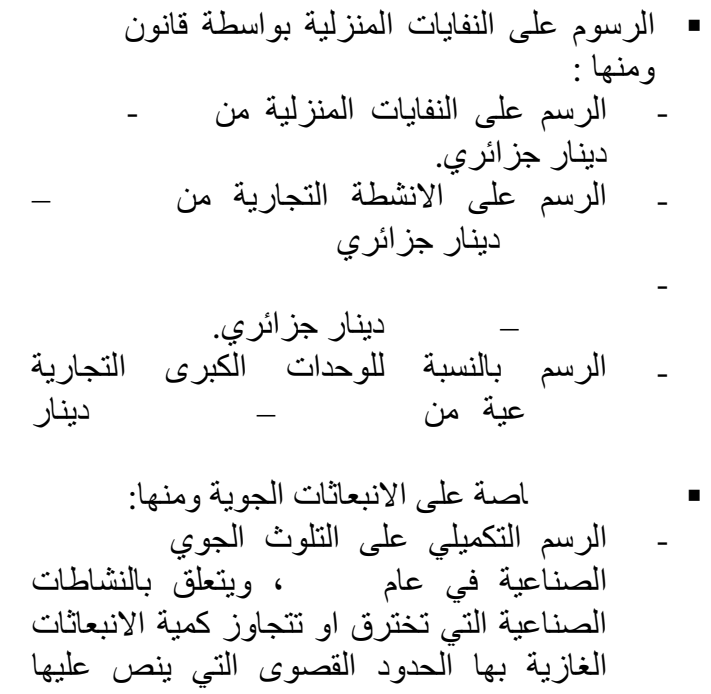

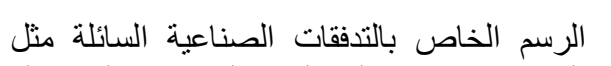

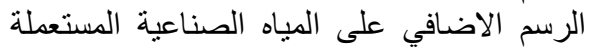

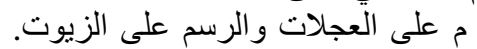

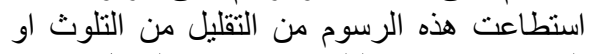

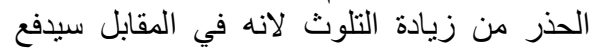

$$
\text { : بعد قانون رقم }
$$

رسوم اضافية.

او قانون مصري موحد لحماية البيئة في مصر فر

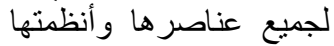
و التذهور ويتوافق ذللك مع الاتجاه التشريعي الدولي الخاص بحماية البيئة.

ويعد هذا القانون بمثابة حماية للبيئة اذ يحتوي على البه اعد القانونية لحماية الهواء ولية والماءية لإئه

اليات اقتصادية لتشجيع الانشطة المختلفة على اتخاذ

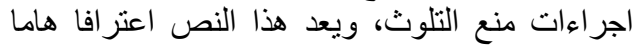

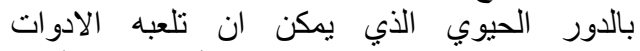
الاقتصادية في تحقيق الإني الدداف السياسات البيئية

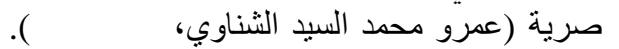

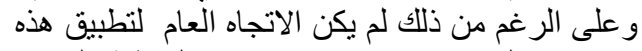

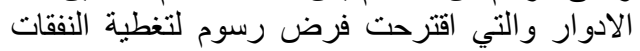
وحقوق الميَّاه

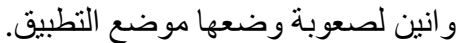

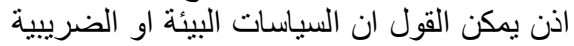

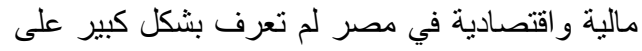

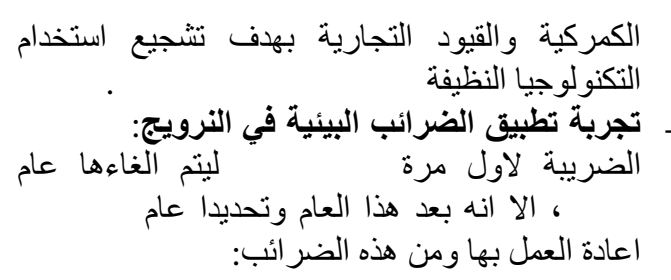

: الدراسات الاقتصادية لتطبيق الضر ائب البيئية: لتي اثبتت كفاءة الضريبة البيئية:

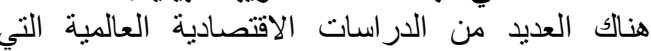

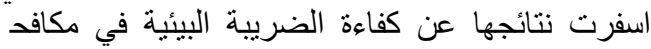

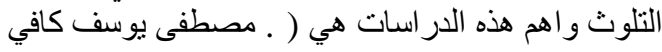

Schultze

استخدام ضريبة التلوث التي تتمثل في تقليل الحاجة التئ

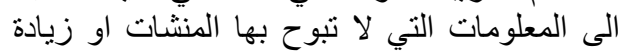

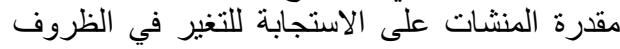

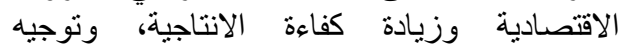
المخترعات نحو المجالات المرغوبة اجتماعيا. Majone

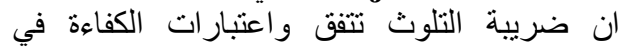
تخصيص الموارد، وتحافظ على المرونة التي تثميز

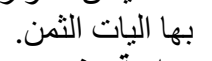

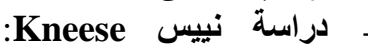

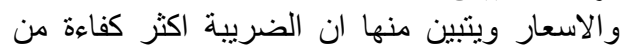

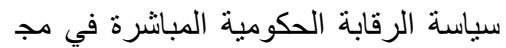
التلوث، كما تبين ان استخدام ضريبة التلوث الته ادى الى الى

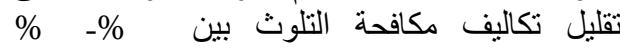
وبالتالي تتحقق وفورات تلتراوح ل Johnson

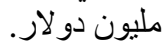

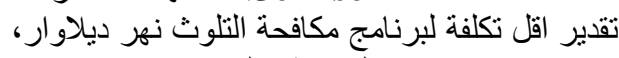

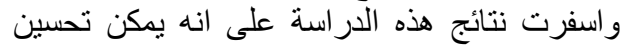
المياه في الثهر باقل تكلفة مدكنة باستخدام نظام التهن

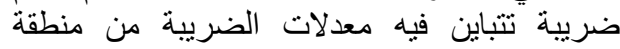

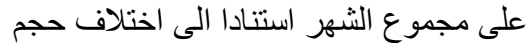
الاضر ار الاخرى بين هذه المناطق. Dorcey

Wisconsin مصانع الورق والمرافق البلدية، وتبين هذه الدراسة

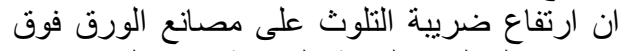

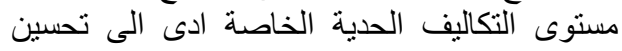

\section{جال تطبيق الضر ائب لحماية البيئة:}

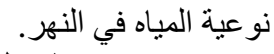

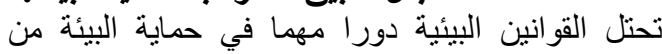
التلوث حيث تعد هذه القوانين ومنها الضرائب البئي البيئية يمتنل لها الجميع لحماية البيئة.

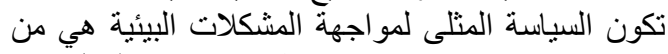
، وقد طبقت بعض المثلات الدول هذه هن

$$
\text { الضر ائب ومنها التجارب التالية: }
$$

الضرائب البيئية في الجزائر: تم تشريع قانون

الملوثة والخطيرة، ولكن لم يطبق هذا القانون الا في

بالنفايات الصلبة ومنها (عبدالقادر عوينات، 
اولت العديد من دول OCDE اهية بالغة لاستخدام

جبائية في اطار البياسات البيئية، حيث لإنيث ب الت على الانبعاثات الملوثة للبيئة.

فنجد ان بعض الدول مثل المانيا، النمسا، سويسرا وسعت الدائرة الجبائية البيئية

الاتاوات كتلك التي تفرض على المركبات الثقيلة

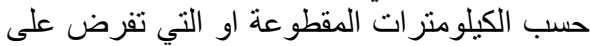
للطرق السريعة او الطرق تضر الطق

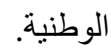
وبشكل عام فان وعاء الضريبة للنفايات في OCDE

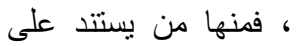

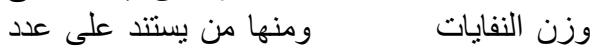

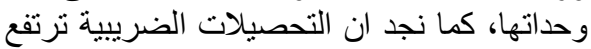

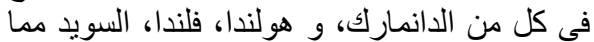
يعكس التطور الكبير للجباية البيئية في هذه الدول النيال

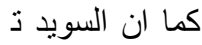

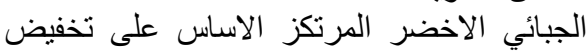

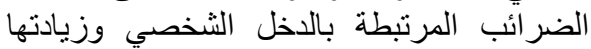
في نفس الوقت على النبعاثات الكربون والكئ والكبريت وألتقليل من استخدام الوقود الاحفوري وبخاص الكربة والكيريت الوقود الذي من يضم مستوى الكبرديت (OCDE,2001,P.67)

: وضع البيئى فى العراق وامكانية تطبيق الضرائب لحماية البيئة.

يعاني ألعراق من الكثير من المشاكل البيئية وذلك بسبب

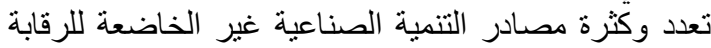

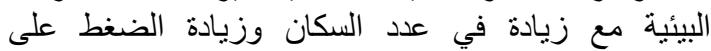

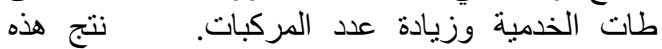
الملوثات زيادة في الكثير من الامر اض التئي التي تصيب الانسان

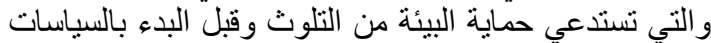
لبيئي يجب الوقوف على حجم التلوث. لئل التيات

قامت وزارة البيئة بنصب محطات لرصد ملوثات الهواء موزعة على عدد من المحافظات حيث يبين لئن المعدلات السنوية لتر اكيز الهايدرو
" ل لمريبة Co على المنتجات البترولية:

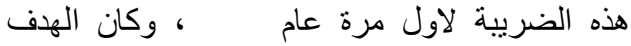
الاساس منها خفض التكاليف المرتبطة بالتقليل من الترن

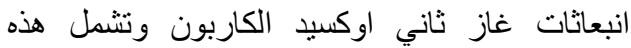
الضريبة الزيت المعدني و النفط و الغاز الطبيعي وغاز CPL وقد ادى فرض الضريبة الى الى

$$
\begin{aligned}
& \text { ت تخفيض مقدار I } \\
& \text { تبنت النرويج نظاما وطنيا } \\
& \% \\
& \text { خاص يرخص التلوث مع ا } \\
& \text { الغازات الدفيئة النرويجية ضمن }
\end{aligned}
$$

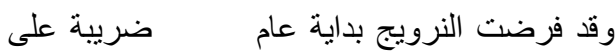

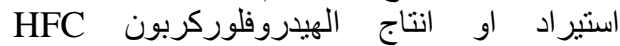
والبيروفليوركربون PFC هذه المو اد عبر تشجيع استخدام غاز اتبرات بديلة اخرى. كما تم ادر اج ضريبة على انبعاثات اكاسيد النتروجين

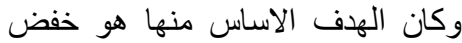
تكاليف و وانبعاثات اكاسيد النتروجين وتنوير التكنولوجيا الني لا تستخدم HFC.

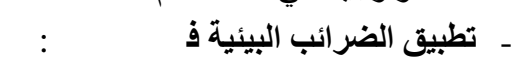

على البيئة ومنها ( Nordic Council of :): (Ministers ,2006,P.200 • الضريبة الطاقوية على الوقود الاحفوري:

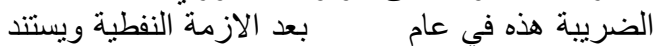

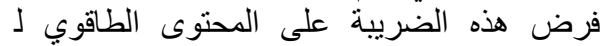
باعتبار ان و عائها الاول ارتبط بالمنتجات النفطية. تم توسيع مجال الضريبة ليشمل الئن

$$
\begin{aligned}
& \text { الغاز الطبيعي عام تاني } \\
& \text { • ضريبة ثاني اكسيد الكاربون: }
\end{aligned}
$$

\begin{tabular}{|c|c|c|c|c|}
\hline ثنائي اوكسيد الكاربون & ثنائي اوكسيد النتروجين & $\begin{array}{c}\text { NMH(1) التركيز جزء بالمليون } \\
\end{array}$ & & \\
\hline 0.04 & 0.04 & 0.774 & 2011 & \multirow{2}{*}{ الوزيرية } \\
\hline 0.35 & 0.04 & 0.828 & 2012 & \\
\hline 0.04 & 0.044 & 0.591 & 2011 & \multirow[t]{2}{*}{ الجادرية } \\
\hline & & 0.600 & 2012 & \\
\hline 0.015 & & 0.077 & 2011 & \multirow[t]{2}{*}{ ابو الخستاوية } \\
\hline 0.011 & & 0.221 & 2012 & \\
\hline 0.014 & & 0.493 & 2011 & \\
\hline 0.027 & & 0.347 & 2012 & \\
\hline
\end{tabular}

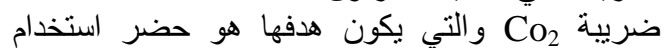

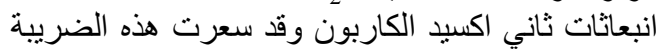
كروته دانماركية / /

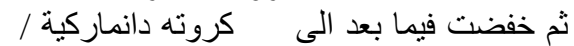

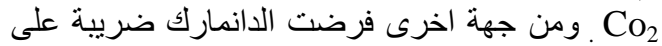
NO $\mathrm{NO}_{\mathrm{x}}$

البيئة.

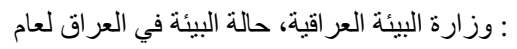


اما ما يتعلق بالغازات الناتجة فان تأثير اتهاسلبية للغاية التانية

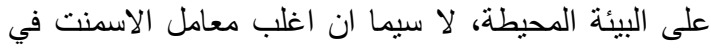
بقية في ابراج

تكرير النفط كوقود و هو من اردأ انواع الوقود مما يؤدي الىى الى

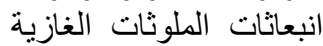
قريبة من الاحياء السكنية خاصة الغية معامل الاسمنت شيدت

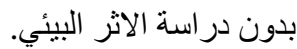

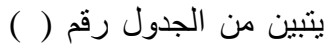

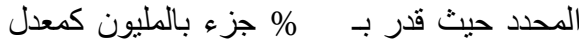

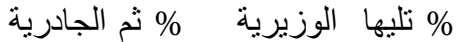

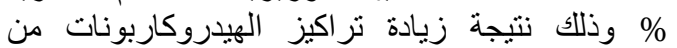

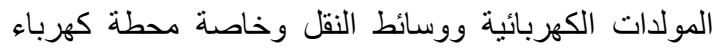
الدورة وكذلك بالنسبة لمحافظة بابل كون المنطة وخلية يكثر فيهة

\begin{tabular}{|c|c|c|c|c|c|}
\hline نسبة الزياة في تركيز المواد & 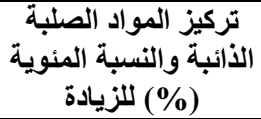 & 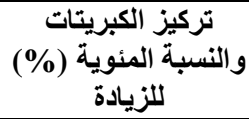 & تركيز العسرة الكلية & تركيز الكلوريدات & \\
\hline منطقة دخول لنهر دجلة الى العي & 254.4 & 45.73 & 209.3 & 26.13 & (T1) \\
\hline 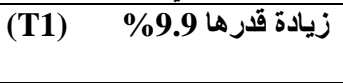 & $\begin{array}{c}279.6 \\
(9.9)\end{array}$ & $\begin{array}{l}57.14 \\
(24.9)\end{array}$ & $\begin{array}{l}228.2 \\
(9.30)\end{array}$ & $\begin{array}{c}30.84 \\
(18.02)\end{array}$ & (T10) \\
\hline $\begin{array}{l}\text { زيادة قدرها 98.6\%) } \\
\text { (T10) }\end{array}$ & $\begin{array}{c}555.4 \\
(118.3) \\
\end{array}$ & $\begin{array}{c}203.4 \\
(344.78)\end{array}$ & $\begin{array}{c}303.7 \\
(45.10)\end{array}$ & $\begin{array}{c}72.3 \\
(176.69)\end{array}$ & (T17) \\
\hline $\begin{array}{l}\text { زيادة قدرها } 17.23 \text { (T17) } \\
\text { (T17) }\end{array}$ & $\begin{array}{c}651.1 \\
(155.9) \\
\end{array}$ & $\begin{array}{c}270.8 \\
(492.17) \\
\end{array}$ & $\begin{array}{c}363.1 \\
(73.48) \\
\end{array}$ & $\begin{array}{c}73.7 \\
(182.05) \\
\end{array}$ & (T24) \\
\hline $\begin{array}{l}\text { زيادة قدرها 18.29) } \\
\text { (T24) }\end{array}$ & $\begin{array}{l}770.2 \\
(202.7)\end{array}$ & $\begin{array}{c}310.7 \\
(576.4)\end{array}$ & $\begin{array}{c}390.2 \\
(86.43)\end{array}$ & $\begin{array}{c}114.6 \\
(338.5)\end{array}$ & (T28) \\
\hline $\begin{array}{l}\text { زيادة قدرها } 23.3 \% \\
\text { (T28) }\end{array}$ & $\begin{array}{c}950 \\
(273.4)\end{array}$ & $\begin{array}{c}306.7 \\
(570.67)\end{array}$ & $\begin{array}{c}476.8 \\
(127.8)\end{array}$ & $\begin{array}{c}302.2 \\
(1056.5)\end{array}$ & (T31) \\
\hline $\begin{array}{l}\text { زيادة قدرها } 2.63 \text { \% } \\
\text { (T31) }\end{array}$ & $\begin{array}{c}975 \\
(383.25)\end{array}$ & $\begin{array}{c}323.3 \\
(606.97)\end{array}$ & $\begin{array}{c}491 \\
(134.59)\end{array}$ & $\begin{array}{c}316.6 \\
(1123.11)\end{array}$ & (T33) \\
\hline $\begin{array}{l}\text { زيادة قدرها } 24.7 \text { \%) } \\
\text { (T33) }\end{array}$ & $\begin{array}{c}1216 \\
(377.98) \\
\end{array}$ & $\begin{array}{c}227.3 \\
(397.04) \\
\end{array}$ & $\begin{array}{c}552.5 \\
(163.97) \\
\end{array}$ & $\begin{array}{c}306 \\
(1123.1) \\
\end{array}$ & (T34) \\
\hline
\end{tabular}

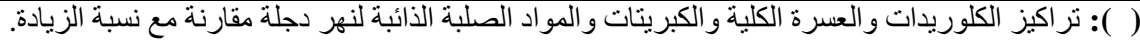

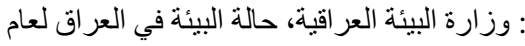

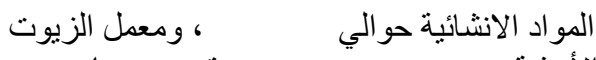

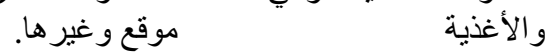

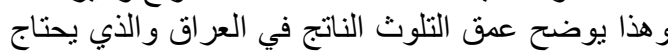
اجر اءات سريعة لتقليل التلوث. تلوث البيئي وتكون من خلال الاتي:

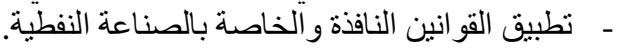

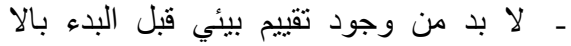
الصناعية. - فرض رسوم وضرائب على المعامل الانشائية

بالمستشفيات وذلك من اجل التعامل الصحيح مع النفايات الطبية. الناتجة عن عمليات

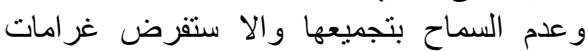
عالية مما يكون رادعا قويا لمنع التلوث.

$$
\text { التنوع البيولوجي. }
$$

اما بالنسبة لمياه الصرف الصحي من اهم مصادر تلوث

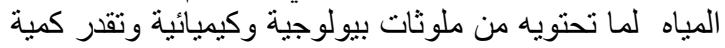

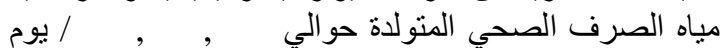

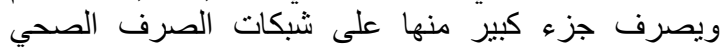
والجزء الاكبر منه يصرف الى المصادر المائية كنهري دجلة

تبلغ عدد محطات معالجة مياه الصرف الصحي لجميع افظات عدا اقليم كردستان محطة ثانوية ومركزية

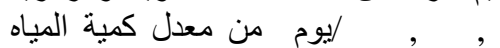
العادمة المتولدة وتبلغُ كمية المياه العادمة المعالجة معنة

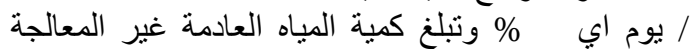

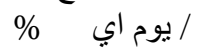

هنالك مخلفات من مصادر السائلة في المصافي

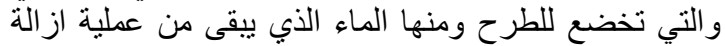
ماء المكثف من العمليات الصناعية.

حيث ان معظم المو اقع ملوثة ومنها قطاع تكرير النفط توجها مواقع ملوثة معظمها من من مصفى المولى الشعيبة ومحطات

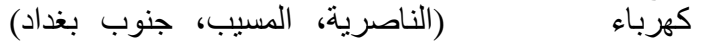
وقطاع البتروكيماويات موقع من معامل الاثيلين المتفرقة بغنة ( ) بوجد في منطقة بيجي الائين 
- الحفاظ على الانظمة البيئية من خلال تفعيل قرار ات البيئية كالضر ائب والرسوم.

البيئي ومشاكله ومع ذلك لم يتم فرض الضر ائب

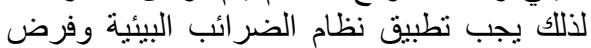

- منح كافة الصلاحيات لوزارة البيئة لحماية البيئة للتاكد من حسن تطبيق هذه الضر ائب.

- - يجب ان يكون هناك وعي بيئي من قبل الافر الن اد

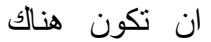

مة للمسؤولين عن الاضرار بالبيئة.

- ضرورة دراسة الحوافز الجبائية في مجال البيئة البئة

واختيار ادو اتها بصفة دقيقة بما يعطي حافز ا كبير الئة

ـ بن حبيب عبدالرزاق، دور الجباية في

ردع وتحفيز المؤسسات الاقتصادية على حماية داية

البيئة من اشكال التلوث: ليلية لنموذج

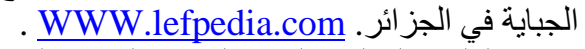

بن طيبة، هوتيه، الملتقى الدولي والني النظام القانوني لحماية البيئة

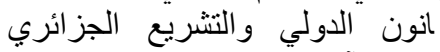

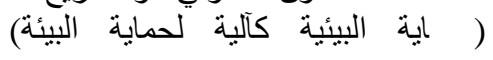

ـ كافي، مصطفى يوسف، اقتصاديات البيئة، دار رسلان،

ـ الربيعي، قاسم كاظم حميد، د. عبدالامير عبدالحسين شياع الهئ

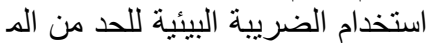

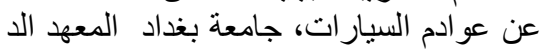

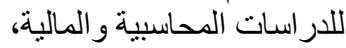

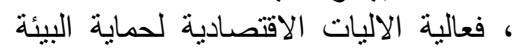

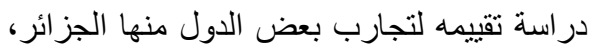

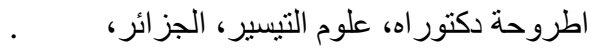

للسياسات الزراعية NAPC، الاقتصاد البيئي

عوينات، عبدالقادر، تحليل الاثار الاقتصادية للمشكلات

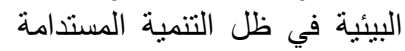

البليدة، كلية العلوم الاقتصادية ولنية وعلوم

الثناوي، عمرو محمد النيد، تقويم يبة كاداة للسياسة

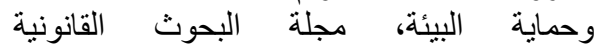

لسياسة الضريبية في كافحة

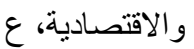

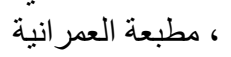

، الهمية تدخل الحكوماتة في حماية البيئة

ل الجباية البيئية، مجلة البيا

وزارة البيئة العراقية، حالة البيئة في

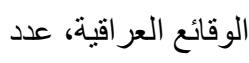

يتضح من خلال العرض السابق ان الوضع في العراق هو

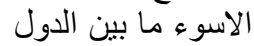

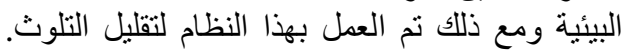

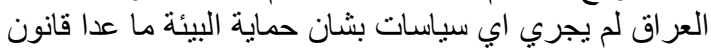

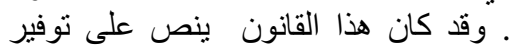

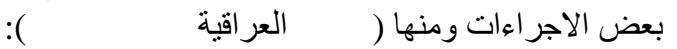

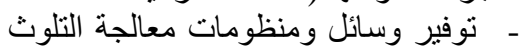
التقنيات الانظف بيئيا.

- توفير اجززة قياس ومر اقبة الملوثات حسب طبقات التها استخدام تقنيات الطاقة المتجددة. وتدوين نتائج القياسات.

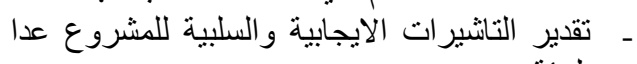
لبيئة. - تقليص المخلفات وتدوير ها او اعادة استخدامها.

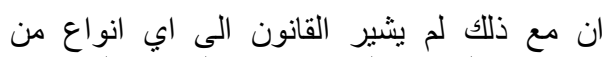

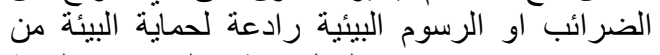
، وهذا يستدعي العمل بنظام الضرائب البئية رائية البينة لحماية البيئة من التلوث. :

- توفير الاليات الاقتصادية لحماية البيئة من اهم ادوات التئ

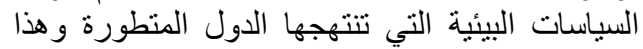

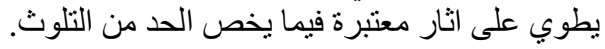

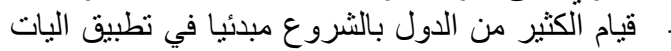

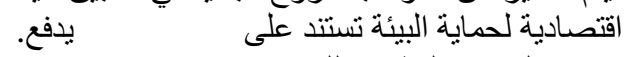

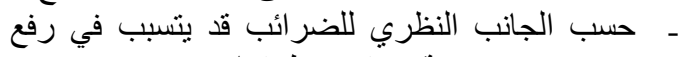
تجة حيث تم تطبيقها في الفي

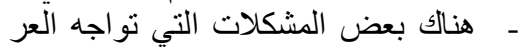

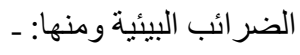

للضريية الحكومية الواجب فرضها على الملى

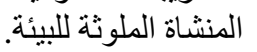

- صعوبة تقدير بعضة لابئة التكاليف الخارجية للانشطة الانتاجية.

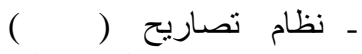

، حيث ان تصاريح التلوث القابلة

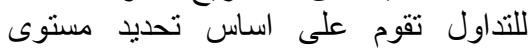

معياري للتلوث وتحديد مقدار للانبعاثات التي تلني

يحدثها الملوثون وتهدف هذه التصاريح الى لئي

- الجباية البيئية هي الاداة ذات الطابع الاقتصادي لتلتوث الأث وحماية البي

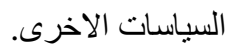
- الضرائب البيئية وسبلة تجبر الافرى اد والثركا

البيئية او ان تتحمل تكاليف نشاطها الضار بالبيئة او ان

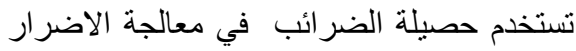

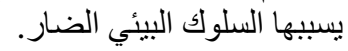

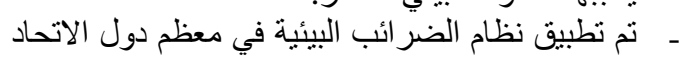
وقد نجحت في تطبيق وتقليل التلوث. 

Kerr,s., Ecological tax Reform, Report prepared for the Ministry of Environment, motu, Economic and public policy research, Newzland, (2001).

Caronline London,environmente instruments economice et fiscaux,libraire general de drioted de jurisprudence, Paris (2001).

OCDE, lestaxesliees al environment dens las pays de locde - problemeset strategies, (2001).

\title{
The role of environmental taxes to reduce pollution \\ Studying The case of some countries the possibility to take advantage of them in Iraq
}

\author{
Muntder Fadil Saad
}

\begin{abstract}
The principle of environmental taxes based on who is happening more environmental damage pays more taxes, as a punishment for the destruction of the environment through various practices. There are kinds of taxes, including the tax on products and waste tax or polluting emissions. Since Iraq is like other countries suffering from environmental problems caused by the long neglect of the environment in all its joints, taxes is one of the ways to reduce the pollutants. However, Iraq doesn't apply this kind of tax or other measures to reduce pollution and protect the environment. The tax imposition in Iraq is facing a lot of difficulties like the difficulty of trying to reach an ideal level of government taxes to protect the environment and others.
\end{abstract}

